\title{
PERFIL DE LA DRA. MARTHA S. DE SEREDAY
}

\author{
PROFILE OF DR. MARTHA S. DE SEREDAY
}

\section{SEMBLANZA DE MI CARRERA PROFESIONAL}

Para comprender las motivaciones que impulsaron mi carrera profesional es necesario ubicarse en el contexto socio-cultural de la época pretecnológica en que crecí, en una ciudad del interior. La mayor de tres hijas de padres muy jóvenes, disfruté de un ambiente familiar propicio, con una madre muy comprometida con los menos favorecidos y excelente dueña de casa; y un padre que pensaba que a las hijas mujeres había que brindarles mayores oportunidades de desarrollar sus aptitudes porque les sería más difícil hacerse en el futuro un lugar en el mundo.

Además no había teléfonos celulares, ni TV satelital, ni Internet. La lectura era la salida intelectual para una mente curiosa. No era habitual el conocimiento de un segundo idioma, salvo en las familias extranjeras.

Argentina era un país ubicado "en el Polo Sur del globo terráqueo" y poco se sabía de nosotros salvo que éramos "el granero del mundo". No era infrecuente recibir cartas dirigidas a "Buenos Aires, Brasil". Las revistas científicas venían de Europa o Estados Unidos en barco y demoraban cuatro semanas en llegar. Los viajes en avión a Nueva York tardaban más de 30 hs con una frecuente parada técnica en Trinidad o Panamá y se cenaba en tierra. Eso sí, tenían sillones muy cómodos.

Esta introducción fue sugerida por mi hijo Carlos Emilio, también médico, y dirigida a los colegas de menos de 45 años. Agradezco a mis cuatro nietos, siempre presentes, que me ayudan a salvar la brecha generacional.

Cursando mi último año de la carrera de Medicina en la UBA (1955) y con dos años de concurrencia a la Guardia del Hospital P. Fiorito, Avellaneda, ya estaba consolidada mi elección profesional, pero no en qué área la desempeñaría: Clínica, Cirugía, Psiquiatría o Investigación; si dedicaría tiempo y esfuerzo a una actividad docente paralela; y cómo armonizaría una vida, que sabía muy comprometida, con mi condición de casada y con un hijo de 1 año, y proyecto de por lo menos otro más.

La Guardia del Hospital me había puesto en claro que debía comenzar con una buena base de Clínica Médica y elegí al Prof. Juan L. Silvestre, Jefe de la Sala de Clínica de Mujeres del mismo como mi mentor. Era muy respetado como semiólogo y docente en el ámbito médico del país.

Al cabo de más de un año, con numerosas charlas con pacientes seguidas de infinitas palpaciones y auscultaciones, muchas recorridas de sala escuchando las opiniones de mis colegas sometidas a los comentarios, correcciones y críticas del Jefe del Servicio y de intensas lecturas, comencé a proyectar mi futuro. En ese momento pude valorar la figura de mi mentor.

El hecho de vivir ambos lejos del Hospital y ser casi vecinos nos acostumbró a viajar juntos cada día y podíamos mantener sin interrupción largas conversaciones sobre Medicina o Sociología (la ubicación del Hospital en un área de inmigrantes de países vecinos y europeos que escapaban a la hambruna de la Segunda Guerra Mundial -de bajos recursos y casi sin educación- nos daba material de sobra).

De mi esposo, centro europeo prácticamente recién llegado al país, aprendí que había otro mundo más allá del Río de la Plata y que en toda formación científica son imprescindibles los idiomas; rescaté los tres aprendidos en el secundario, y comencé a viajar a Estados Unidos.

Un día el Prof. Silvestre trajo el tema de la necesidad de empezar a pensar en una especialidad y comenzamos a ahondar en dos o tres posibles inclinándose él a la Endocrinología porque era un terreno que comenzaba a desarrollarse rápidamente y por su amistad y respeto por el Prof. E. del Castillo, entonces uno de los tres líderes de la especialidad en Argentina (1957). Hice con él una pasantía de dos años conservando mi concurrencia de una vez por semana al Hospital Fiorito de 
donde traía dudas, errores y pacientes, situación que me resultaba muy desafiante y competitiva.

Al incrementarse la proporción de diabéticos que atendíamos surgió la necesidad de complementar esta pasantía con una concurrencia al Servicio de Nutrición del Hospital Rawson a cargo del Prof. Pedro Landabure, donde tuve el privilegio de conocer, frecuentar y disfrutar más tarde de la amistad de los Dres. Néstor Serantes, Saúl Senderey y Bernardo Nusimovich, quien "apadrinó" mi formación ulterior en el extranjero.

A él quiero dedicarle un momento de reflexión. Nusimovich era una persona muy inteligente, con una memoria colosal y una innata capacidad para las relaciones público-profesionales y con amigos en todo el mundillo científico. En interminables charlas de café armamos a través de tres años un programa de visitas de un mes de duración a distintos centros científicos norteamericanos con un referente de su círculo, que me distinguió con sus enseñanzas. Dos de ellos merecen un párrafo por su persistencia en el tiempo: el Dr. Robert Grenblatt de Augusta, Ga. que dedicó sus investigaciones al ovario poliquístico y la menopausia, y el Dr. Leo Krall de la Joslin Clinic, Boston Mass., excelente docente de postgrado. Sus respectivos hijos aún son amigos de nuestra familia.

Con ese bagaje participé durante tres años como docente en temas de Endocrinología en los cursos libres sobre Semiología y Clínica Propedéutica a cargo del Prof. Silvestre (1958-60). Más tarde acompañaría al Dr. Nusimovich en los cursos del Hospital Rawson (1968) y comenzarían a convocarme para reuniones científicas en el interior del país y en el exterior.

En 1971 accedí a la Jefatura del Servicio de Endocrinología del Hospital Fiorito en un Concurso Abierto por Oposición y mi primera acción fue incluir en la denominación del mismo "y Diabetes". El término Nutrición se reservaba para el Servicio de Alimentación.

Y entonces comenzó la segunda parte de mi formación, cuando en la primera reunión con las autoridades y frente a mis demandas de equipamiento del sector diabetes me preguntaron: "¿Para atender a cuántas personas y con qué características?". Por respuesta, total silencio de mi parte y salí a contar diabéticos. No fue fácil porque no había información estadística de base poblacional ni protocolos para obtenerla.

Para hacer el Primer Estudio de Prevalencia de
Diabetes (DM) tuve que recurrir a la Organización Panamericana de la Salud (OPS), culminando con una publicación sobre el tema cuya metodología fue luego recomendada por la Oficina para Latinoamérica y mi designación como Asesora Temporal de la misma (1984). Mi actividad allí incluyó más tarde la organización de Centros de Atención Primaria en México y El Caribe.

En 1987, en una reunión del European Diabetes Epidemiology Group (EDEG) en París, presenté algunos resultados de mis investigaciones y asesorías, a raíz de lo cual fui invitada a incorporarme al grupo. Éste se reúne anualmente en diferentes países de la región y durante las discusiones con los más destacados epidemiólogos mundiales surgen nuevos modelos de estudio y de análisis de las observaciones fisiopatológicas, clínicas y terapéuticas de esta nueva epidemia, como se la denomina en la actualidad a la DM.

Ante el avance de la epidemiología como herramienta científica surgió la necesidad de incluirla en la formación de los médicos clínicos y especialistas. Con el Dr. Manuel L Martí organizamos en 1993 el Primer Curso Internacional de Epidemiología sobre el modelo del homónimo de Cambridge. La convocatoria generó gran expectativa entre los que atendían diabéticos en Latinoamérica. Hubo numerosos pedidos ulteriores de bibliografía y de asesorías, y como corolario surgió el Grupo Latinoamericano de Epidemiología de la Diabetes -GLED- (1994) integrado por profesionales del área, el cual continúa hasta la fecha su labor docente con seis cursos ya realizados en distintos países de Latinoamérica. El próximo año será en Perú.

En 1999 se realizó en Bs. As. una exitosa reunión científica conjunta GLED-EDEG. Los europeos se sorprendieron con las presentaciones de los especialistas peruanos sobre diabetes en la altura, de los paraguayos y brasileros sobre diabetes en la selva, de las tasas de mortalidad por diabetes en Centro América etc., material casi imposible de publicar entonces en Europa o Estados Unidos, no sólo por la dificultad idiomática.

Con los Dres. R Bolaño y C González realizamos durante tres años cursillos informativos de tres días en el interior del país sobre Conceptos Básicos de Estadística y Epidemiología (1990) que fueron de gran utilidad para analizar los resultados obtenidos en los estudios efectuados por los grupos locales y para interpretar la literatura médica. 
Junto con los médicos y el bioquímico del Servicio del Hospital Fiorito realizamos y publicamos, además de los estudios sobre prevalencia e incidencia de DM tipo 1 y 2, otros sobre las características de las complicaciones crónicas en los recién diagnosticados, sobre los valores de la PTGO en embarazadas sin diabetes (del que participó el Dr. Peter Bennett del NIH USA y consultor del EDEG), entre otros.

Participé como integrante del Comité Académico coordinado por el Dr. Juan José Gagliardino en la programación, ejecución y evaluación de dos programas de educación dirigidos al personal médico y no médico del país: Programa de Capacitación del Médico Generalista (PROCAMEG, 1997-2004) capacitando 500 médicos de distintos capítulos de la SAD y evaluando impacto clínico y metabólico de sus pacientes (900) antes y después del curso. Y Capacitación en Diabetes del Equipo de Salud (CADIEQ 2004-2005) del cual se desarrollaron 25 cursos en las seis especialidades programadas (Asistente Social, Educador en Diabetes, Profesor de Educación Física, Enfermería, Nutrición, Podología) con la formación de 230 especialistas integrados en 54 equipos con un médico con formación diabetológica actuando como coordinador.

Miembro del Cómite Académico de Diabetes y Factores de Riesgo Cardiovascular (DIFAR), curso semi presencial (online) desarrollado a través del Departamento de Graduados de la Facultad de Ciencias Médicas de la Universidad de la Plata y de Indiana University (Estados Unidos), coordinado por el Dr. JJ Gagliardino y dirigidos a médicos de primer contacto (2004 hasta la fecha).
Con el Dr. Martí y nuestro grupo del Hospital Fiorito obtuvimos en 1998 el premio Mariano R Castex de la Academia Nacional de Medicina con la compilación de los Estudios Descriptivos sobre DM realizados: "Aspectos clínicos de la epidemiología de la DM". Fue la primera vez que una investigación epidemiológica ganó allí un premio clínico.

En 2003 fui invitada a pronunciar en París la Conferencia Houssay en el marco del Congreso de la Federación Internacional de Diabetes. Mi tema elegido fue "Epidemiología" al que subtitulé "De la siembra a la cosecha" con el convencimiento que en ambos desempeños, ciencia y cultivos, es necesario identificar el objetivo de la intervención y respetar las distintas etapas del desarrollo del protocolo adecuado.

Cuando comencé a organizar el contenido de este artículo me di cuenta que en mi vida profesional había seguido los mismos lineamientos, sea por intuición o por personalidad.

Entre líneas quedan las experiencias positivas y negativas del diario quehacer médico; la camaradería de los viajes al exterior con los colegas argentinos para asistir a reuniones y congresos, y los nuevos amigos de aquí y de allá que ellos generaron; los contrastes entre la rudeza del mercado popular y el Teatro Bolshoi en Moscú o entre las elegantes tiendas de París y Roma, y los souks del norte de África; la satisfacción por las distinciones y las tristezas por los pacientes a los que no pudimos ayudar.

Al concluir hago mías las palabras del escritor chileno Pablo Neruda, quien tituló a su libro autobiográfico: "Confieso que he vivido". 